\title{
THE PREVALENCE OF DYSLIPIDEMIA IN PATIENTS ATTENDING THE POST KIDNEY TRANSPLANT CLINIC AT ST PAUL'S HOSPITAL
}

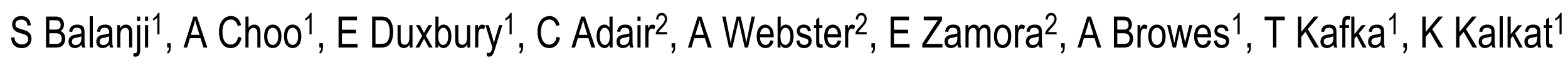 \\ ${ }^{1} \mathrm{UBC}$ Dietetics Program \\ 2Providence Health Care
}

Introduction:

Renal Transplant Recipients (RTR) are at high risk of dyslipidemia. Dyslipidemia is defined by the Canadian Cardiovascular Society as a low-density lipoprotein $(\mathrm{LDL})$ value $>2.0 \mathrm{mmol} / \mathrm{L}$ in high risk groups. Elevated LDL has been shown to be closely tied to Cardiovascular Disease (CVD). The prevalence of dyslipidemia in the St Paul's Hospital (SPH) RTR population has not been previously investigated.

\section{Objectives:}

To describe demographic data and biochemical markers at 1 Year Post Transplant (YPT) and the percentage of RTR with dyslipidemia over 1, 2, and 5 YPT.

\section{Methods:}

A retrospective chart review was completed for SPH RTR over 18 years old who received transplants between January 1st, 1997 and July 31st, 2018. Descriptive statistics were generated for the sample at 1,2 , and 5YPT.

\section{Results:}

The inclusion criteria was met by 796 RTR (M:61\%, F:39\%) and among this sample, 185 RTR (23\%) had missing LDL values 1YPT. At 1YPT, the proportion of RTR with dyslipidemia was $79 \%(n=611)$ and declined slightly in 2 and 5YPT (78 \%, n=577, 77\%, n=412). Females (83\%, $n=257,81 \% n=247,76 \% n=176$ ) have a higher prevalence in each year than males $(75 \% n=384,75 \% n=330,75 \% n=236)$. Of our two largest groups, White ethnicity $(81 \% \quad n=303, \quad 80 \% \quad n=252, \quad 79 \% \quad n=220)$ demonstrated higher prevalence than East Asian individuals (74\% $n=145,72 \% n=145,72 \% n=104$ ) across all 3-time points.
Implications and Conclusions:

This study confirms the importance of monitoring LDL values in RTR. It may also have identified a need to improve consistency in blood lipid testing in order to identify dyslipidemia earlier on post-transplant. This and future research could lead to a clinical decision-making pathway to help medical professionals determine appropriate and timely dyslipidemia interventions.

Characteristics of Renal Transplant Recipients at 1 Year Post Transplant, $n=796$

Sex, $n$

Ethnicities, $\mathrm{n}$

$\begin{array}{cc}\text { White } & 395 \\ \text { East Asian } & 191 \\ \text { Asian Indian } & 75 \\ \text { Filipino } & 62 \\ \text { North American Indian } & 33 \\ \text { Middle Eastern/Arabian } & 13 \\ \text { Black } & 12 \\ \text { Other/Unknown/Multiracial } & 9 \\ \text { Latin American } & 6\end{array}$

Age, (years) mean +/- SD

$51.11+/-13.64$

Blood lipids, (mmol/L) mean +/-SD

$\begin{array}{ll}\text { TC, } n=651 & 5.01+/-1.24 \\ \text { HDL, } n=632 & 1.38+/-0.46 \\ \text { LDL, } n=611 & 2.81+/-0.95 \\ \text { Triglycerides, } n=643 & 1.75+/-1.24\end{array}$

\begin{tabular}{|c|c|c|c|c|c|}
\hline Univariate Analysis of Dem & phic an & al Parameters of RTRs with & pider & $2 \mathrm{mmol} / \mathrm{L})$ at 1,2 , and $5 \mathrm{Y}$ & ost $\mathrm{Tr}$ \\
\hline $\begin{array}{rr} & \text { 1 Year Post Trar } \\
\text { Age (years) } & \% \mathrm{R} \\
\text { dys! }\end{array}$ & $\begin{array}{l}\text { ant } \\
\text { with } \\
\text { emia }\end{array}$ & \begin{tabular}{|rr}
\multicolumn{2}{|c}{2 Years Post Tra } \\
Age (years) & $\%$ R \\
dys!
\end{tabular} & $\begin{array}{l}\text { ant } \\
\text { with } \\
\text { emia }\end{array}$ & \begin{tabular}{|r}
\multicolumn{2}{|c}{5 Years Post T } \\
Age (years)
\end{tabular} & $\begin{array}{l}\text { lant } \\
\text { with } \\
\text { emia }\end{array}$ \\
\hline $18-34.9 n=77$ & $73 \%$ & $18-34.9 n=84$ & $80 \%$ & $18-34.9 n=63$ & $78 \%$ \\
\hline $35-44.9 n=102$ & $81 \%$ & $35-44.9 n=98$ & $74 \%$ & $35-44.9 n=75$ & $81 \%$ \\
\hline $45-54.9 n=173$ & $83 \%$ & $45-54.9 n=167$ & $84 \%$ & $45-54.9 n=133$ & $80 \%$ \\
\hline $55-64.9 n=149$ & $83 \%$ & $55-64.9 n=141$ & $76 \%$ & $55-64.9 n=93$ & $73 \%$ \\
\hline 65 and up $n=110$ & $67 \%$ & 65 and up $n=87$ & $76 \%$ & 65 and up $n=44$ & $66 \%$ \\
\hline Sex & & Sex & & Sex & \\
\hline Female $n=257$ & $83 \%$ & Female $n=247$ & $81 \%$ & Female $n=176$ & $76 \%$ \\
\hline Male $n=354$ & $75 \%$ & Male $n=330$ & $75 \%$ & Male $n=236$ & $75 \%$ \\
\hline Ethnicities & & Ethnicities & & Ethnicities & \\
\hline Middle Eastern/Arabian $n=5$ & $100 \%$ & Black $n=10$ & $90 \%$ & Latin American $n=1$ & $100 \%$ \\
\hline Other/Unknown/Multiracii $n=7$ & $86 \%$ & Other/Unknown/Multiracii $n=7$ & $86 \%$ & Asian Indian $n=26$ & $92 \%$ \\
\hline White $n=303$ & $81 \%$ & Asian Indian $n=53$ & $83 \%$ & White $n=220$ & $79 \%$ \\
\hline Black $n=10$ & $80 \%$ & White $n=252$ & $80 \%$ & Filipino $n=27$ & $78 \%$ \\
\hline Asian Indian n=63 & $79 \%$ & Middle Eastern/Arabian $n=5$ & $80 \%$ & Middle Eastern/Arabian $n=4$ & $75 \%$ \\
\hline Latin American $n=4$ & $75 \%$ & Filipino $n=29$ & $79 \%$ & East Asian $n=104$ & $72 \%$ \\
\hline East Asian $n=145$ & $74 \%$ & East Asian $n=145$ & $72 \%$ & Black $n=7$ & $71 \%$ \\
\hline Filipino $n=47$ & $70 \%$ & North American Indian $n=21$ & $71 \%$ & Other/Unknown/Multira $n=3$ & $67 \%$ \\
\hline North American Indian $n=15$ & $65 \%$ & Latin American $n=5$ & $60 \%$ & North American Indian $n=15$ & $60 \%$ \\
\hline BMI (kg/m^²) & & BMI $\left(\mathrm{kg} / \mathrm{m}^{\wedge} 2\right)$ & & BMI (kg/m^2) & \\
\hline$<18 n=11$ & $50 \%$ & $<18 n=14$ & $71 \%$ & $<18 n=14$ & $43 \%$ \\
\hline $19-25 n=306$ & $79 \%$ & $19-25 n=248$ & $81 \%$ & $19-25 n=182$ & $83 \%$ \\
\hline $26-30 n=144$ & $81 \%$ & $26-30 n=164$ & $77 \%$ & $26-30 n=105$ & $75 \%$ \\
\hline $31+n=111$ & $79 \%$ & $31+n=92$ & $73 \%$ & $31+n=52$ & $83 \%$ \\
\hline Creatinine (mmol/L) & & Creatinine (mmol/L) & & Creatinine (mmol/L) & \\
\hline Lower $(40-110) n=276$ & & Lower $(40-110) n=272$ & & Lower $(40-110) n=204$ & $77 \%$ \\
\hline Moderate $(111-200) n=310$ & $76 \%$ & Moderate $(111-200)_{n}=278$ & $77 \%$ & Moderate $(111-200) n=190$ & $76 \%$ \\
\hline Higher $(>200) n=25$ & $68 \%$ & Higher $(>200) n=26$ & $73 \%$ & Higher $(>200) n=17$ & $82 \%$ \\
\hline $\begin{array}{l}\text { Total Sample } \\
n=611\end{array}$ & $79 \%$ & $\begin{array}{l}\text { Total Sample } \\
n=577\end{array}$ & $78 \%$ & $\begin{array}{l}\text { Total Sample } \\
n=412\end{array}$ & $77 \%$ \\
\hline
\end{tabular}

